\title{
LOWER SEMICONTINUITY OF PARAMETRIC INTEGRALS
}

\author{
BY \\ EDWARD SILVERMAN $\left.{ }^{1}\right)$
}

\begin{abstract}
It has been known for a long time that the usual two-dimensional parametric integrals in three-space are lower semicontinuous with respect to uniform convergence. In an earlier paper we saw that an easy argument extends this result to all parametric integrals generated by simply-convex integrands, with no restrictions on the dimension of the surfaces or the containing space. By using these techniques again, and generalizing to surfaces a result concerning convergent sequences of closed curves we show that a parametric integral generated by a parametric integrand which is convex in the Jacobians is lower semicontinuous with respect to uniform convergence provided all of the functions lie in a bounded subset of the Sobolev space $H_{s}^{1}$ where $s+1$ exceeds the dimension of the parametric integral.
\end{abstract}

We apply the technique used to study parametric integrals generated by simply-convex integrands to show that parametric integrals generated by integrands which are convex in the Jacobians are lower semicontinuous on bounded subsets of suitable Sobolev spaces.

We are interested in the lower semicontinuity result of [Mc-1], [C-1], [M-2]. The problems which they consider are either of codimension one, in which case the integrands are automatically simply-convex or there is an explicit additional restriction which is, except for name, simplicity. In [S-3] we obtained this result and were able to drop additional technical hypotheses which had been imposed. Using that result, the representation theorem from [S-1] and the characterization of simplicity from $\lfloor\mathrm{S}-2\rfloor$ we get, I think, the easiest existence theorem to the classical two-dimensional parametric variational problem. (This method also sometimes gives smoothness properties of the solution.) In this paper we drop the condition on simplicity, but must now adjoin the additional hypothesis that all of the functions involved lie in a bounded subset of a suitable Sobolev space in order to retrieve the lower semicontinuity theorem. We intend to apply this result to get an existence theorem for the two-dimensional problem without the hypothesis of simplicity.

We start off by extending to more than two-dimensions a result concerning

Received by the editors January 3, 1972.

AMS (MOS) subject classifications (1970). Primary 49A50; Secondary 26A63, 28 A75.

Key words and phrases. Topological index, essential multiplicity, Peano area, Lebesgue area, Sobolev space.

(1) This research was supported in part by the National Science Foundation under Grant 6075-50-13955. 
the limit of the integral of the topological indices corresponding to a sequence of closed curves in the plane which converge uniformly and are uniformly bounded in length.

Let $e_{1}, \ldots, e_{\nu}$ be an orthonormal basis for $R^{\nu}$ and let $R^{\nu}$ be oriented by $e_{1} \wedge \ldots \wedge e_{\nu}$. Let $S$ be the unit sphere in $R^{\nu}$. If $\omega_{1} \in S$ there exist $\omega_{2}$, $\cdots, w_{\nu} \in S$ such that $\left\{\omega_{i} \mid i=1, \ldots, \nu\right\}$ is a basis for $R^{\nu}$ and $\omega_{1} \wedge \ldots \wedge \omega_{\nu}=$ $e_{1} \wedge \cdots \wedge e_{\nu}$. Let $R_{\omega_{1}}^{\nu}$ be the vector space spanned by $\omega_{2}, \ldots, \omega_{\nu}$ which is oriented by $\omega_{2} \wedge \cdots \wedge \omega_{\nu}$. If $\omega \in S$ let $\pi_{\omega}$ be the orthogonal projection of $R^{\nu}$ onto $R_{\omega}^{\nu}$ If $p \in R^{\nu}$ let $p_{\omega}=\{p+\lambda \omega \mid \lambda \geq 0\}$.

If $\alpha$ is a $\nu$-form over $R^{\nu}$ then $\alpha=\lambda e_{1} \wedge \ldots \wedge e_{\nu}$ for some real number $\lambda$. Let $\operatorname{sgn} \alpha=\operatorname{sgn} \lambda$.

Let $\mathcal{P}$ be the collection of all bounded oriented polyhedral regions $J$ in $R^{\nu}$ such that $\partial J$ is the union of a finite number of $(\nu-1)$-dimensional oriented boxes each of which is orthogonal to a coordinate axis. If $J \in \mathcal{P}, X \in C\left(J, R^{\nu}\right)$ and $p \in R^{\nu}$, let $O(p, X, J)$ be the topological index of $p$ with respect to $X$ and $J$. If $x=X \mid \partial J$ let $M\left(\pi_{\omega} p, \pi_{\omega} x, \partial J\right)$ be the essential multiplicity of $\pi_{\omega} p$ with respect to $\pi_{\omega} x$ and $\partial J[F]$.

Theorem 1. Let $X \in C\left(J, R^{\nu}\right), x=X \mid \partial J$ and $\omega \in S$. Then there exists a subset $\mathcal{E}(x, \omega)$ of $R_{\omega}^{\nu}$ having measure zero such that

$$
2|O(p, X, J)| \leq M\left(\pi_{\omega} p, \pi_{\omega} x, \partial j\right)
$$

if $\pi_{\omega} p \notin \mathcal{E}(x, \omega)$.

Proof. Let $\left\{a_{0}, a_{1}, \ldots, a_{\nu-1}\right\}$ be the vertices of an oriented $(\nu-1)$ simplex $\delta$ in $R^{\nu}$ and $y$ be linear (affine) on $\delta$ into $R^{\nu}$. Suppose, in addition, that $y$ is light (in this case $y$ is a homeomorphism of $\delta$ onto $y(\delta)$ ). Let

$$
y^{*}(\delta)=\left(y\left(a_{1}\right)-y\left(a_{0}\right)\right) \wedge \cdots \wedge\left(y\left(a_{\nu-1}\right)-y\left(a_{0}\right)\right) .
$$

It is easy to verify that $y^{*}(\delta)$ does not depend upon the particular representation of $\delta$ which we chose. If $p \in R^{\nu}$ let us write $\left\{p_{\omega}, y(\delta)\right\}$ if $p_{\omega}$ does not intersect the boundary of $y(\delta)$ relative to its containing $(\nu-1)$-plane. If $\left\{p_{\omega}, y(\delta)\right\}$ then $[\mathrm{A}-\mathrm{H}, \mathrm{p} .412]$

$$
\phi\left(p_{\omega}, y(\delta)\right)= \begin{cases}0 & \text { if } p_{\omega} \text { and } y(\delta) \text { are disjoint } \\ \operatorname{sgn}\left(p_{\omega} \wedge y^{*}(\delta)\right) & \text { otherwise. }\end{cases}
$$

If $p \notin y(\delta)$ then either $\phi\left(p_{\omega}, y(\delta)\right)=0$ or $\phi\left(p_{-}, y(\delta)\right)=0$ so that

$$
\left|O\left(\pi_{\omega} p, \pi_{\omega} y, \delta\right)\right|=\left|\phi\left(p_{\omega}, y(\delta)\right)\right|+\left|\phi\left(p_{-}, y(\delta)\right)\right| \text {. }
$$

Now suppose that $x=X \mid \partial J$ is light and quasilinear. Let $\Delta$ be the collection of oriented $(\nu-1)$-simplexes of linearity of $x$. Then [A-H, p. 458-459] 


$$
O(p, X, J)=\sum_{\delta \in \Delta} \phi\left(p_{\omega}, x(\delta)\right)
$$

provided $p \notin X(\partial J)$ and $\left\{p_{\omega}, x(\delta)\right\}$ for all $\delta \in \Delta$. Hence

$$
2|O(p, x, J)| \leq \sum_{\delta \in \Delta}\left|O\left(\pi_{\omega} p, \pi_{\omega} x, \delta\right)\right| \leq M\left(\pi_{\omega} p, \pi_{\omega} x, \partial J\right)
$$

provided $\left\{p_{\omega}, x(\delta)\right\}$ and $\left\{p_{-}, x(\delta)\right\}$ for all $\delta \in \Delta$.

Let us return to the general case. By $[F]$ there exist light quasilinear $y_{n}$ $\rightarrow \pi_{\omega}^{x}$ in $C\left(\partial J, R_{\omega}^{\nu}\right)$ such that

$$
\int_{R_{\omega}^{\nu}} M\left(q, y_{n}, \partial J\right) d q \rightarrow \int_{R_{\omega}^{\nu}} M\left(q, \pi_{\omega} x, \partial J\right) d q .
$$

Let $\Delta_{n}$ be the collection of simplexes of linearity of $y_{n}$. We define $x_{n}$ on $\partial J$ as follows: $x_{n} \mid \delta$ is linear for each $\delta \in \Delta_{n}$ and $x_{n}(u)=y_{n}(u)+(x(u) \cdot \omega) \omega$ if $u$ is a vertex of $\delta$. Then $x_{n}$ is light and quasilinear. Also $x_{n} \rightarrow x$ in $C\left(\partial J, R^{\nu}\right)$ and there exist $X_{n} \in C\left(J, R^{\nu}\right)$ such that $X_{n} \mid \partial J=x_{n}$ and $X_{n} \rightarrow X$ in $C\left(J, R^{\mathcal{Y}}\right)$. If $\pi_{\omega} p$ does not belong to the union of countably many subsets of $R_{\omega}^{\nu}$, each having measure zero, then

$$
\begin{aligned}
& 2|O(p, X, J)| \leq 2 \lim \inf \left|O\left(p, x_{n}, J\right)\right| \\
& \quad \leq \lim \inf M\left(\pi_{\omega} p, \pi_{\omega} x_{n}, \partial J\right)=\lim \inf M\left(\pi_{\omega} p, y_{n}, \partial J\right) .
\end{aligned}
$$

By $(*), \lim \inf M\left(q, y_{n}, \partial J\right)=M\left(q, \pi_{\omega} x, \partial J\right)$ for almost all $q \in R_{\omega}^{\nu}$. Hence if $\pi_{\omega} p$ does not belong to a set $\mathcal{E}(x, \omega)$ in $R_{\omega}^{\nu}$ which has measure zero, then

$$
2|O(p, X, J)| \leq M\left(\pi_{\omega} p, \pi_{\omega} x, \partial J\right) .
$$

If $X|\partial J=Y| \partial J=x$ then $O(p, X, J)=O(p, Y, J)$ and we can denote the common value by $o(p, x, \partial J)$. In this notation the conclusion of the last theorem is $2|o(p, x, \partial J)| \leq M\left(\pi_{\omega} p, \pi_{\omega} x, \partial J\right)$.

Let $P$ denote Peano area.

Theorem 2. Let $J \in \mathcal{P}, x \in C\left(\partial J, R^{\nu}\right)$ and $\omega \in S$. Then

$$
\int_{R_{\omega}^{\nu}} M\left(q, \pi_{\omega} x, \partial J\right) d q \leq P(x) .
$$

Proof. By the definition of Peano area and $[F]$,

$$
P(x) \geq P\left(\pi_{\omega} x\right)=\int_{R_{\omega}^{\nu}} M\left(q, \pi_{\omega} x, \partial J\right) d q .
$$

If $\alpha=1, \ldots, \nu$ let $R_{\alpha}^{\nu}=R_{\omega}^{\nu}$ where $\omega=e_{\alpha}$.

The next theorem is related to the result of [L-W].

Theorem 3. If $f_{\alpha} \in L^{\nu-1}\left(R_{\alpha}^{\nu}\right)$ then 


$$
\left\|\prod_{a=1}^{\nu} f_{a} \circ \pi_{a}\right\| \leq \prod_{a=1}^{\nu}\left\|f_{a}\right\|_{\nu-1}
$$

Proof. The inequality obviously holds if $\nu=2$. Let us assume that it holds if $\nu=n$. Let $g_{\alpha}=\left|f_{\alpha} \circ \pi_{\alpha}\right|, b_{\alpha}=g_{\alpha}^{n}, d u_{1}=d u^{2} \cdots d u^{n}, d u_{n}=d u^{1} \cdots d u^{n-1}$ and $d u_{a}=d u^{1} \ldots d u^{\underline{a}-1} d u^{a+1} \ldots d u^{n}$ for $1<\alpha<n$. Then

$$
\begin{aligned}
\int \prod_{a=1}^{n+1} g_{a} & =\int g_{n+1}\left[\int \prod_{a=1}^{n} g_{a} d u^{n+1}\right] d u^{1} \cdots d u^{n} \\
& \leq\left\|f_{n+1}\right\|_{n}\left\{\int\left[\prod_{a=1}^{n} g_{a} d u^{n+1}\right]^{n /(n-1)} d u^{1} \cdots d u^{n}\right\}^{(n-1) / n} \\
& \leq\left\|f_{n+1}\right\|_{n}\left\{\int \prod_{a=1}^{n}\left\{\int b_{a} d u^{n+1}\right\}^{1 /(n-1)} d u^{1} \cdots d u^{n}\right\}^{(n-1) / n} \\
& \leq\left\|f_{n+1}\right\|_{n}\left\{\prod_{a=1}^{n}\left[\int\left\{b_{a} d u^{n+1}\right\} d u_{a}\right]^{1 /(n-1)}\right\}^{(n-1) / n}=\prod_{a=1}^{n+1}\left\|f_{a}\right\|_{n}
\end{aligned}
$$

Theorem 4. Let $J \in \mathcal{P}$. Suppose that $x$ and $x_{n}$ are in $C\left(\partial J, R^{\nu}\right)$ with $x_{n} \rightarrow x$ and $\left\{P\left(x_{n}\right)\right\}$ uniformly bounded. If $\mid$ range $x \mid=0$ then

$$
\lim \int_{R^{\nu}} o\left(p, x_{n}, \partial J\right) d p=\int_{R^{\nu}} o(p, x, \partial J) d p
$$

and

$$
\lim \int_{R^{\nu}}\left|o\left(p, x_{n}, \partial J\right)\right| d p=\lim \int_{R^{\nu}}|o(p, x, \partial J)| d p
$$

Proof. We repeat, with slight modifications, the argument in [C-3, Pp. 9596] for $\nu=2$.

There exists a cube $Q$ in $R^{\nu}$ which contains range $x$ and range $x_{n}$ for all $n$. If $v \in R_{a}^{\nu}$ let $S_{n}^{a}(v)=M\left(v, \pi_{a}^{x} n^{\prime} \partial J\right) / 2$. By Theorem 1 ,

$$
\left|o\left(p, x_{n}, \dot{\partial} J\right)\right| \leq M\left(\pi_{a} p, \pi_{a} x_{n}, \partial J\right) / 2
$$

provided $\pi_{a} p$. does not belong to a set $Z_{a} \subset R_{a}^{\nu}$ with measure $Z_{a}=0$. For each positive integer $m$ let $I_{n m}^{\alpha}=\left\{v \in R_{\alpha}^{\nu} \mid S_{n}^{a}(v) \geq m\right\}$. Thus

$$
m\left|I_{n m}^{a}\right| \leq \int_{I_{n m}^{a}} S_{n}^{a}(v) d v \leq \int_{R_{a}^{\nu}} M\left(\nu, \pi_{a} x_{n}, \partial J\right) d v
$$

so that $\left|I_{n m}^{\alpha}\right| \leq M m^{-1}$ for some $M>0$.

Let

$$
I_{n m}=\left\{p \in R^{\nu}|| o\left(p, x_{n}, \partial J\right) \mid \geq m\right\}
$$


and

$$
J_{n m}=\left\{p \in R^{\nu}|| o\left(p, x_{n}, \partial J\right) \mid=m\right\} .
$$

If $p \in I_{n m}$ then $m \leq S_{n}^{\alpha}\left(p_{a}\right)$. Thus $\pi_{a} p \in I_{n m}^{\alpha}$. If $i_{n m}^{\alpha}$ and $i_{n m}$ are the characteristic functions of $I_{n m}^{a}$ and $I_{n m}$, respectively, then

$$
i_{n m} \leq \prod_{a=1}^{\nu} i_{n m}^{a} \circ \pi_{a^{*}}
$$

By Theorem 3, $\left|I_{n m}\right| \leq \Pi_{a=1}^{\nu}\left\|i_{n m}^{a}\right\|_{\nu-1}$ so that $\left|I_{n m}\right| \leq\left(M m^{-1}\right)^{\nu /(\nu-1)}$. In addition, $I_{n m}=\bigcup_{r \geq m} J_{n r}$ so that $\left|I_{n m}\right|=\Sigma_{r \geq m}\left|J_{n r}\right|$. Thus, if $T_{n m}=$ $\int_{I_{n m}}\left|o\left(p, x_{n}, \partial J\right)\right| d p$, then

$$
\begin{aligned}
T_{n m} & =\sum_{r \geq m}\left|J_{n r}\right|=m \sum_{r \geq m}\left|J_{n r}\right|+\sum_{r>m}(r-m)\left|J_{n r}\right| \\
& =m\left|I_{n m}\right|+\sum_{r>m}\left|J_{n r}\right| \sum_{s=m+1}^{r} 1=m\left|I_{n m}\right|+\sum_{s>m} \sum_{r \geq s}\left|J_{n r}\right|=m\left|I_{n m}\right|+\sum_{s>m}\left|I_{n s}\right|
\end{aligned}
$$

so that

$$
T_{n m}<m\left(M m^{-1}\right)^{\nu /(\nu-1)}+\sum_{r>m}\left(M r^{-1}\right)^{\nu /(\nu-1)} .
$$

Now let $\epsilon>0$. There exists $m$ such that $T_{n m}<\epsilon / 2$. Let $\eta=\epsilon /(2 m)$ and $b$ be a measurable subset of $Q$ with $|b|<\eta$. Then $b=b^{\prime} \cup b^{\prime \prime}$ where $b^{\prime}=$ $\left(Q \sim I_{n m}\right) \cap b$ and $b^{\prime \prime}=I_{n m} \cap b$. Thus $\int_{b}\left|o\left(p, x_{n}, \partial J\right)\right| d p<\epsilon$ for all $n$, and the theorem follows.

Let $N \geq \nu$ and $E=R^{N}$.

We need the following well-known result.

Theorem 5. Let $Q$ be the unit cube in $R^{\nu-1}$ and let $s>\nu-1$. There exists $K>0$ depending only upon $s$ and $\nu$ such that if $x \in C(\rho Q, E) \cap$ $H_{s}^{1}($ Int $\rho Q, E)$ then

$$
\text { diain } x(\rho 2) \leq K \varphi^{1-(\nu-1) / s}\left\|x^{\prime}\right\|_{s} .
$$

The proof consists of approximating $x$ by smooth functions $y$ and estimating the average distance from $y(p)$ to $y(q)$ for some fixed point $p \in \rho Q$, where $q$ is an arbitrary point in $\rho Q$.

If $A \subset E$ and $t>0$ let $H^{t}(A)$ be the Hausdorff $t$-dimensional measure of $A$.

Corollary. If $x \in C(Q, E) \cap H_{s}^{1}($ Int $Q, E)$, then $H^{t}(x(Q))=0$ for all $t>\nu-1$.

Proof. We can suppose that $t=s$. Let $\epsilon>0$. Take $n$ so large that $K n^{(\nu-1-s) / s}\left\|x^{\prime}\right\|_{s}<\epsilon / 2$. Let $Q$ be subdivided into congruent cubes $Q_{i}$, 
$i=1, \ldots, n^{\nu-1}$. Let $p_{i} \in Q_{i}$ and let $B$ be the open unit ball in $E$. Then $x\left(p_{i}\right)+K n^{(\nu-1-s) / s}\left\|x^{\prime}\right\|_{s, Q_{i}} B$ is a ball which contains $x\left(Q_{i}\right)$ and has diameter less than $\epsilon$. Furthermore,

$$
\sum_{i}\left[K n^{(\nu-1-s) / s}\left\|x^{\prime}\right\|_{s, Q}\right]_{i}^{s}=K^{s} n^{\nu-1-s}\left\|x^{\prime}\right\|_{s}^{s}
$$

and this last term can be made arbitrarily small by taking $n$ big enough.

If $G$ is a bounded open set in $R^{\nu}$ and if $r \in R$ let

$$
G^{a}(r)=\left\{u \in G \mid u^{a}=r\right\}, \quad \alpha=1, \ldots, \nu .
$$

If $x$ is a function defined on $G$ let $x(\alpha, r)=x \mid G^{\alpha}(r)$.

The proof of the following lemma appears in [M-2, p. 118].

Lemma 1. Let $G$ be a bounded open set in $R^{\nu}$ and let $\left(a^{a}, b^{a}\right)$ be the projection of $G$ on the atb-axis. Suppose $x_{m} \in H_{s}^{1}(G, E)$ for $m=1,2, \ldots$, and that $\left\{\left\|x_{m}\right\|_{s}^{1}\right\}$ is uniformly bounded. Then there exist

(i) $D>d>0$,

(ii) infinite subsets $N_{n}$ of the natural numbers with $N_{n} \subset N_{n+1}$,

(iii) $\left\{t_{n, i}^{\alpha} \mid i=1, \ldots, 2^{n}+1\right\} \subset\left[a^{\alpha}, b^{\alpha}\right], t_{n, i-1}^{\alpha}<t_{n, i}^{\alpha}$ for $i \neq 1, t_{n, 1}^{\alpha}=a^{\alpha}$, and $t_{n, 2^{n}+1}^{\alpha}=b^{\alpha}$,

(iv) $d \leq\left(t_{n, i}^{\alpha}-t_{n, i-1}^{\alpha}\right) /\left(b^{\alpha}-a^{\alpha}\right) \leq D$ for $i \neq 1$, such that $\left\{\left\|x_{m}\left(a, t_{n, i}^{\alpha}\right)\right\|_{s}^{1} \mid\right.$ $\left.m \in N_{n}\right\}$ is uniformly bounded if $1 \neq i \neq 2^{n}+1$.

From [G-Z] and the fact that Peano area is not bigger than Lebesgue area, it follows that

$$
\left\{P\left(x_{m}\left(\alpha, t_{n, i}^{a}\right)\right) \mid m \in N_{n}\right\}
$$

is uniformly bounded if $1 \neq i \neq 2^{n}+1$.

Now let $\psi$ be continuous and nonnegative on $E \times T$ where $T=\wedge^{\nu} E$. If $a \in E$ let $\psi_{a}$ be defined on $T$ by $\psi_{a}(\sigma)=\psi(a, \sigma)$ for all $\sigma \in T$ and suppose that $\psi_{a}$ is convex and that $\psi_{a}(\lambda \sigma)=\lambda \psi_{a}(\sigma)$ for all $\lambda>0$ and $\sigma \in T$. Let

$$
A=\{a \in E \mid \psi(a, \sigma)>0 \text { for all } \sigma \neq 0\}
$$

and let

$$
\theta(\zeta, a)=\max \{[\sigma, \zeta] \mid \psi(a, \sigma) \leq 1\}
$$

for all $a \in A$ and $\zeta \in T^{\prime} \approx \Lambda^{\nu} E^{\prime}$. Finally, let

$$
r(\zeta, a)= \begin{cases}1 / \theta(\zeta, a) & \text { if } \zeta \neq 0 \text { and } a \in A, \\ 0 & \text { otherwise. }\end{cases}
$$

Then $\tau_{\zeta}$ is lower semicontinuous, where $\tau_{\zeta}(a)=\tau\left(\zeta_{0} a\right)$. In fact, $\tau_{\zeta} \mid A$ is continuous, [S-3]. Furthermore, $\psi(a, \sigma)=\sup _{\zeta} \tau(\zeta, a)[\sigma, \zeta]$. 
If $\eta \in E^{\prime \nu}$ let $\eta^{*} \in L\left(E, R^{\nu}\right)$ be defined by

$$
\eta^{*} b=\sum_{i=1}^{\nu}\left[b, \eta^{i}\right] e_{i}
$$

where, as usual, $e_{i} \in R^{\nu}, e_{i}^{j}=\delta_{i}^{j}$. Let $G$ be an open subset of $R^{\nu}$ and let $C=$ $C(G, E)$, with the topology of uniform convergence on compact subsets of $G$. If $x \in C$ and $\eta \in E^{\prime \nu}$ then $\eta^{*} x$ is a flat map from $G$ to $R^{\nu}$. We saw, [S-3], that if $J$ is a polyhedron contained in $G$ and if $\xi \in E^{\prime \nu}$ with $\xi_{1} \wedge \ldots \wedge \xi_{\nu}=$ $\eta_{1} \wedge \cdots \wedge \eta_{\nu}$, then

$$
\int_{R^{\nu}} K^{ \pm}\left(q, \xi^{*} x, J\right) d q=\int_{R^{\nu}} K^{ \pm}\left(r, \eta^{*} x, J\right) d r
$$

where the integrands are the positive or negative essential multiplicity functions. Thus if $\zeta$ is a simple element of $T^{\prime}$ we can define

$$
K^{ \pm}(\zeta, x, J)=\int_{R^{\nu}} K^{ \pm}\left(q, \xi^{*} x, J\right) d q
$$

where $\zeta=\xi^{1} \wedge \ldots \wedge \xi^{\nu}$. Similarly, we can define

$$
A(\zeta, x, J)=\int_{R^{\nu}} O\left(p, \xi^{*} x, J\right) d p .
$$

If $\left|\xi^{*} x(\partial J)\right|=0$ then

$$
A(\zeta, x, J)=\mathscr{\varkappa}^{+}(\zeta, x, J)-\mathcal{K}^{-}(\zeta, x, J)
$$

If $P(x)$ is finite then the Lebesgue derivatives of $A(\zeta, x, \cdot), \mathcal{K}^{+}(\zeta, x, \cdot)$ and $K^{-}(\zeta, x,$.$) are J_{e}\left(\xi^{*} x\right)$, the essential generalized Jacobian of $\xi^{*} x, J_{e}^{+}\left(\xi^{*} x\right)$ and $J_{e}^{-}\left(\xi^{*} x\right)$, and

$$
\left|J_{e}\left(\xi^{*} x\right)\right|=J_{e}^{+}\left(\xi^{*} x\right)+J_{e}^{-}\left(\xi^{*} x\right)
$$

almost everywhere [R-R, pp. 258-261].

Now suppose $\zeta$ is an arbitrary element of $T^{\prime}$. Let 9 be the collection of all increasing $\nu$-termed subsequence of the integers $1,2, \ldots, N$. Then $\zeta=$ $\Sigma_{\lambda \in g} \zeta_{\lambda} e^{\lambda}$ for suitable $\zeta_{\lambda} \in R$ where $e^{\lambda}=e^{\lambda_{1}} \wedge \ldots \wedge e^{\lambda} \nu$ if $\lambda=\left\{\lambda_{1}\right.$, $\left.\lambda_{2}, \ldots, \lambda_{\nu}\right\}$. We define

$$
A(\zeta, x, J)=\sum_{\lambda \in \mathfrak{g}} \zeta_{\lambda} A\left(e^{\lambda}, x, J\right) .
$$

Let $\mathcal{O}=\{J \in \mathscr{P} \mid J \subset G\}$. If $x \in C, \zeta \in T^{\prime}$ and $J \in \mathcal{O}$ let

$$
H(x, J, \zeta)=\min \{r(\zeta, x(p)) \mid p \in J\},
$$


so that [S-3], $H$ is lower semicontinuous on $C$ for fixed $J$ and $\zeta$.

If $x \in C(G, E)$ and if $P(x)<\infty$ let

$$
P_{\psi}(x)=\sup _{\sigma} \sum_{J \in \sigma} \sup _{\zeta} H(x, J, \zeta) A(\zeta, x, J)
$$

where $\sigma$ is a finite subset of $\mathcal{C}$ whose distinct elements are nonoverlapping.

We recall that $P(x)=P_{\psi_{0}}(x)$ where $\psi_{0}(a, \sigma)=\|\sigma\|$ for all $a \in E$ and $\sigma \in T$.

Lemma 2. Let $\left\{t_{n, i}^{a}\right\}$ satisfy (i)-(iv) of Lemma 1 and let $(9)$ be the set of all those $J \in \mathcal{P}$ whose faces lie on $G^{a}\left(t_{n, i}^{a}\right), 1 \neq i \neq 2^{n}+1$. Suppose that $H^{\nu}(x(\partial J))=0$ for some $x \in C(G, E)$ and all $J \in \mathbb{C}$. Let $\tau$ be a finite set of nonoverlapping polybedra of li. If $P(x)<\infty$, then

$$
P_{\psi}(x)=\sup _{\tau} \sum_{J \in \tau} \sup _{\zeta} H(x, J, \zeta) A(\zeta, x, J) .
$$

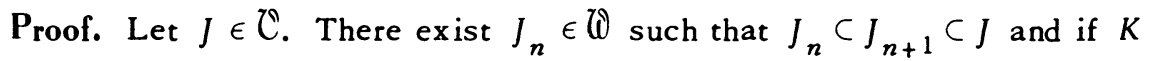
is a compact subset of Int $J$, then $K \subset J_{n}$ for sufficiently large $n$. Hence if $\xi \in E^{\prime \nu}$ then

$$
\left|O\left(p, \xi^{*} x, J_{n}\right)\right| \leq M\left(p, \xi^{*} x, J\right) \text { and } \lim _{n} O\left(p, \xi^{*} x, J_{n}\right)=O\left(p, \xi^{*} x, J\right) \text { a.e. }
$$

Thus $A\left(\zeta, x, J_{n}\right) \rightarrow A(\zeta, x, J)$ for all $\zeta \in T^{\prime}$ and the lemma follows.

Theorem 6. Let $s>\nu-1$ and let $x_{n} \in C(G, E) \cap H_{s}^{1}(G, E)$ with $P\left(x_{n}\right)<$ $\infty$ and $\left\{\left\|x_{n}\right\|_{s}^{1}\right\}$ uniformly bounded. If $x_{n} \rightarrow x$ in $C(G, E)$ and if $P(x)<\infty$ then $P_{\psi}(x) \leq \lim \inf P_{\psi}\left(x_{n}\right)$.

Proof. Let us use the notation of Lemmas 1 and 2. A subsequence $x_{n_{k}}$ converges weakly to $y$ in $H_{s}^{1}(G, E)$. Thus $x_{n_{k}}$ converges weakly to $y$ in $L_{s}(G, E)$. Since $x_{n_{k}}$ converges weakly to $x$ in $L_{s}\left(G^{\prime}, E\right)$ for any $G^{\prime}$ with $G^{\prime} \subset G$, thus $x=y$ so that $x \in H_{s}^{1}(G, E)$ and $\|x\|_{s}^{1} \leq \lim$ inf $\left\|x_{n_{k}}\right\|_{s}^{1}$. Similarly,

$$
x\left(\alpha, t_{n, i}^{\alpha}\right) \in H_{s}^{1}\left(G^{a}\left(t_{n, i}^{\alpha}\right)\right)
$$

and $\left\|x\left(a, t_{n, i}^{a}\right)\right\|_{s}^{1}$ is finite for each $(\alpha, n, i)$ provided $1 \neq i \neq 2^{n}+1$. Let $\epsilon>0$. There exists a finite subset $\tau$. of $l^{i}$ with $|\tau|$ elements, whose distinct elements are nonoverlapping, and $\zeta_{J} \in T^{\prime}$ for each $J \in \tau$ such that

$$
P_{\psi}(x)-\epsilon<\sum_{J \in \tau} H\left(x, J, \zeta_{J}\right) A\left(\zeta_{J}, x, J\right)
$$

There is no loss in generality in supposing that $\lim P_{\psi}\left(x_{n}\right)$ exists. There exists a subsequence $\left\{x_{n_{k}}\right\}$ such that $\left\{\left\|x_{n_{k}} \mid \partial J\right\|_{s}^{1}\right\}$ is uniformly bounded for each $J \in \tau$. Since $s>\nu-1$, the Lebesgue area of $x_{n_{k}} \mid \partial J$ is given by the usual formula $[G-Z]$. Hence $\left\{P\left(x_{n_{k}}\right) \mid \partial J\right\}$ is uniformly bounded for each 
$J \in \tau$. If $\xi \in E^{\prime \nu}$ then $\xi^{*} x_{n_{k}}$ and $\xi^{*} x$ are all continuous on $\partial J, \xi^{*} x_{n_{k}}$ converges uniformly to $\xi^{*} x$ on $\partial J,\left\{P\left(\xi^{*} x_{n_{k}}\right) \mid \partial J\right\}$ is uniformly bounded and, by the corollary to Theorem $5,\left|\xi^{*} x(\partial J)\right| \leq|x(\partial J)|=0$. Thus by Theorem 4 and the definition of $A$,

$$
\lim _{k \rightarrow \infty} A\left(\zeta, x_{n_{k}}, J\right)=A(\zeta, x, J)
$$

where $\zeta=\xi_{1} \wedge \ldots \wedge \xi_{\nu}$. Hence there exists $M$ such that

$$
\sum_{J \in T} H\left(\zeta_{J}, J, x\right) A\left(\zeta_{J}, x, J\right)<\sum_{J \in T} H\left(\zeta_{J}, J, x_{n_{k}}\right) A\left(\zeta_{J}, x_{n_{k}}, J\right)+\epsilon
$$

for all $k>M$. Thus $P_{\psi}(x)-\epsilon<P_{\psi}\left(x_{n_{k}}\right)+\epsilon$ for all $k>M$.

If $x$ is differentiable, let $\bigwedge x^{\prime}=x_{1} \wedge \ldots \wedge x_{\nu}$. Thus

$$
\psi\left(x, \wedge x^{\prime}\right)=\sup _{\zeta \in T} r(x, \zeta)\left[\wedge x^{\prime}, \zeta\right] .
$$

If $\lambda=\left\{\lambda_{1}, \ldots, \lambda_{\nu}\right\} \in g$ let $x^{\lambda}=\left(x^{\lambda_{1}}, \ldots, x^{\lambda} \nu\right)$. If $\zeta=\Sigma \zeta_{\lambda} e^{\lambda}$ then $\left[\wedge x^{\prime}, \zeta\right]=$ $\Sigma \zeta_{\lambda} J\left(x^{\lambda}\right)$. If $x$ is eBV we define

$$
\psi\left(x, / x^{\prime}\right)=\sup _{\zeta \in T}, r(x, \zeta) \sum_{\lambda \in \mathfrak{g}} \zeta_{\lambda} J_{e}\left(x^{\lambda}\right)
$$

and let $I_{\psi}(x)=\int_{G} \psi\left(x, \bigwedge x^{\prime}\right)$.

Theorem 7. If $x$ is eBV then $P_{\psi}(x) \geq I_{\psi}(x)$ and the equality bolds if $x$ is $e A C$.

We use the proof of [S-3]. If $\phi(J)=P_{\psi}(x \mid J)$ then $\phi$ is superadditive so that $D P_{\psi}(x)$ exists almost everywhere. Let $\zeta \in T^{\prime}$. Then

$$
D P_{\psi}(x) \geq \tau(x, \zeta) \sum_{\lambda \in \mathbb{S}} \zeta_{\lambda} J_{e}^{\left(x^{\lambda}\right)}
$$

almost everywhere. Hence $P_{\psi}(x) \geq I_{\psi}(x)$. Now suppose that $x$ is eAC. If $\zeta \epsilon$ $T^{\prime}$ then

$$
\psi\left(x, \wedge^{\prime}\right) \geq r(x, \zeta) \sum_{\lambda \in \mathfrak{g}} \zeta_{\lambda} J_{e}^{\left(x^{\lambda}\right)}
$$

so that

$$
I_{\psi}(x \mid J) \geq H(x, \zeta, J) \sum_{\lambda \in \Phi} \zeta_{\lambda} A\left(e^{\lambda}, x, J\right)=H(x, \zeta, J) A(\zeta, x, J)
$$

so that $I_{\psi}(x) \geq P_{\psi}(x)$.

Theorem 8. If $x \in H_{\nu}^{1}(G, E) \cap C(G, E)$ then $x$ is eAC.

Proof. If $\xi \in E^{\prime \nu}$ let $J\left(\xi^{*} x\right)$ be the ordinary Jacobian of $\xi^{*} x$. By [G-Z], $L\left(\xi^{*} x\right)=I_{\psi_{0}}\left(\xi^{*} x\right)<\infty$, where $L$ denotes Lebesgue area. Since $P \leq L$, it follows that $x$ is eBV. By [R-R, p. 351], $J\left(\xi^{*} x\right)=J_{e}\left(\xi^{*} x\right)$ a.e. if $\xi^{*} x$ has a weak 
total differential almost everywhere. By $[\mathrm{G}-\mathrm{Z}], \xi^{*} x$ has a weak total differential almost everywhere. According to [R-R, p. 261], $I_{\psi_{0}}\left(\xi^{*} x\right) \leq P\left(\xi^{*} x\right)$ with equality holding if and only if $\xi^{*} x$ is eAC. The theorem follows since $I_{\psi_{0}}\left(\xi^{*} x\right) \leq P\left(\xi^{*} x\right)$ $\leq L\left(\xi^{*} x\right)=I_{\psi_{0}}\left(\xi^{*} x\right)$.

\section{REFERENCES}

[A-H] P. S. Aleksandrov and H. Hopf, Topologie, Springer, Berlin, 1935.

[C-1] Lamberto Cesari, Condizioni necessarie per la semicontinuità degli integrali sopra una superficie in forma parametrica, Ann. Mat. Pura Appl. (4) 29 (1949), 199-224. MR 12, 86.

[C-2] - An existence theorem of calculus of variations for integrals on parametric surfaces, Amer. J. Math. 74 (1952), 265-295. MR 14, 292.

[C-3] - Surface area, Ann. of Math. Studies, no. 35, Princeton Univ. Press, Princeton, N. J., 1956. MR 17, 596.

[D] J. M. Danskin, Jr. , On the existence of minimizing surfaces in parametric double integral problems in the calculus of variations, Riv. Mat. Univ. Parma 3 (1952), 43-63. MR 14, 292.

[F] H. Federer, Essential multiplicity and Lebesgue area, Proc. Nat. Acad. Sci. U.S.A. 34 (1948), 611-616. MR 10, 361.

[G-Z] C. Goffman and W. P. Ziemer, Higher dimensional mappings for which the area formula holds, Ann. of Math. (2) 92 (1970), 482-488. MR $42 \# 6166$.

[L-W] L. H. Loomis and $\mathrm{H}$. Whitney, An inequality related to the isoperimetric inequality, Bull. Amer. Math. Soc. 55 (1949), 961-962. MR 11, 166.

[Mc] E. J. McShane, On the semicontinuity of double integrals in the calculus of variations, Ann. of Math. 33 (1932), 460-484.

[M-1] C. B. Morrey, Jr., The parametric variational problem for double integrals, Comm. Pure Appl. Math. 14 (1961), 569-575. MR 24 \#A1638.

[M-2] - Multiple integrals in the calculus of variations, Die Grundlehren der math. Wissenschaften, Band 130, Springer-Verlag, New York, 1966. MR 34 \#2380.

[R-R] T. Radó and P. Reichelderfer, Continuous transformations in analysis. With an introduction to algebraic topology, Die Grundlehren der math. Wissenschaften in Einzeldarstellugen mit bedanderer Berücksichtigung der Anwendungsgebiete, Band 75, Springer-Verlag, Berlin, 1955. MR 18, 115.

[Si] A. G. Sigalov, Two-dimensional problems of the calculus of variations, Uspehi Mat. Nauk 6 (1951), no. 2 (42), 16-101; English transl, Amer. Math. Soc. Transl. (1) 6 (1962), 27-146. MR 13, 257; 1139.

[S-1] Edward Silverman, A problem of least area, Pacific J. Math. 14 (1964), 309331. MR $30 \# 1229$.

[S-2] —_, Simple areas, Pacific J. Math. 15 (1965), 299-303. MR 31 \#1584.

[S-3] —_ A sufficient condition for the lower semicontinuity of parametric integrals, Träns. Amer. Math. Soc. 167 (1972), 465-469.

DIVISION OF MATHEMATICAL SCIENCES, PURDUE UNIVERSITY, WEST LAFAYETTE, INDIANA 47907 